QUARTERLY OF APPLIED MATHEMATICS

VOLUME LXVII, NUMBER 4

DECEMBER 2009, PAGES 781-791

S 0033-569X(09)01147-5

Article electronically published on May 27, 2009

\title{
ORBITAL STABILITY OF STANDING WAVE SOLUTION FOR A QUASILINEAR SCHRÖDINGER EQUATION
}

\author{
BY
}

BOLING GUO (Institute of Applied Physics and Computational Mathematics, P.O. Box 8009, Beijing 100088, People's Republic of China)

AND

JIANQING CHEN (School of Mathematics and Computer Science, Fujian Normal University, Fuzhou, 350007, People's Republic of China)

Abstract. Via minimization arguments and the Concentration Compactness Principle, we prove the orbital stability of standing wave solutions for a class of quasilinear Schrödinger equation arising from physics.

1. Introduction. This paper is motivated by recent interest in the following type of quasilinear Schrödinger equation:

$$
i \partial_{t} z=-\Delta z+V(x) z-k\left(\Delta\left(|z|^{2}\right)\right) z-\theta|z|^{p-2} z,
$$

where $i$ is the imaginary unit, $p>2$ and $k, \theta \in \mathbb{R}_{+}, \Delta z=\sum_{j=1}^{N} \partial^{2} z / \partial x_{j}^{2}$ the standard Laplacian operator. $z:=z(x, t): \mathbb{R}^{N} \times \mathbb{R}_{+} \rightarrow \mathbb{C}$ is a complex-valued function.

Problems of this kind arise naturally from various domains of mathematical physics and have been derived as models of several physical phenomena in the theory of superfluid films and in dissipative quantum mechanics (see e.g. Kurihura [8], Nakamura [14]). For more physical motivations and more references dealing with applications, we refer the interested readers to Lange et al. 9], Poppenberg et al. 15] and the references therein.

Via critical point theory, finding a standing wave of the form $z(x, t)=e^{i \mu t} u(x)$ of problem (1.1) is equivalent to solving the following elliptic equation:

$$
-\Delta u+(V(x)+\mu) u-k\left(\Delta\left(|u|^{2}\right)\right) u-\theta|u|^{p-2} u=0 .
$$

Under the basic assumption of $\inf _{x \in \mathbb{R}^{N}} V(x)+\mu>0$, the existence of nontrivial solutions of (1.2) has been studied in the last two decades. Some of them are due to Strauss [17] and Rabinowitz [16] in the case of $k=0$ and Ambrosetti-Liu-Wang-Wang [2, 11, 12, 13.

Received September 17, 2008.

2000 Mathematics Subject Classification. Primary 35Q55, 35A15, 35B35.

Key words and phrases. Standing wave solution, orbital stability, quasilinear Schrödinger equation.

The second author is supported by the National Natural Sciences Foundation of China.

E-mail address: jqchen@fjnu.edu.cn

(C)2009 Brown University 
in the case of $k \neq 0$ with some additional assumptions on $V(x)$ and $p$. Using the basic function space $H^{s}\left(\mathbb{R}^{N}\right)=W^{s, 2}\left(\mathbb{R}^{N}\right)$ and setting

$$
X=\left\{\left.u \in H^{1}\left(\mathbb{R}^{N}\right)\left|\int_{\mathbb{R}^{N}}\right| u\right|^{2}|\nabla u|^{2} d x<\infty, \quad \int V(x)|u|^{2}<\infty\right\},
$$

we can define functionals:

$$
\begin{gathered}
F_{1}(u)=\frac{1}{2} \int\left(|\nabla u|^{2}+V(x)|u|^{2}\right), \quad F_{2}(u)=\frac{1}{2} \int|u|^{2}, \\
F_{3}(u)=\left.\left.\frac{1}{4} \int|\nabla| u\right|^{2}\right|^{2}, \quad F_{4}(u)=\frac{1}{p} \int|u|^{p}, \\
F_{5}(u)=F_{1}(u)+\mu F_{2}(u)+k F_{3}(u)-\theta F_{4}(u), \\
F_{6}(u)=\int\left(|\nabla u|^{2}+(V(x)+\mu)|u|^{2}+\left.\left.k|\nabla| u\right|^{2}\right|^{2}-\theta|u|^{p}\right) .
\end{gathered}
$$

Problem (1.2) has a formal variational structure. For any $\phi \in \mathcal{D}\left(\mathbb{R}^{N}\right), F_{j}, j=1, \ldots, 6$, have directional derivatives at $u$ in the direction $\phi$, denoted by $\left\langle F_{j}^{\prime}(u), \phi\right\rangle$. We say that $u$ is a weak solution of (1.2) if and only if for any $\phi \in \mathcal{D}\left(\mathbb{R}^{N}\right)$ and some $\mu, \theta$, it follows that

$$
\left\langle F_{1}^{\prime}(u)+\mu F_{2}^{\prime}(u)+k F_{3}^{\prime}(u)-\theta F_{4}^{\prime}(u), \phi\right\rangle=0 .
$$

Up to now, we have three methods to study the existence of standing wave solutions for problems of this kind.

Method I: Study the minimization problem

$$
m_{I}=\inf \left\{F_{1}(u)+\mu F_{2}(u)+k F_{3}(u) \mid u \in X, F_{4}(u)=\lambda_{I}>0\right\} .
$$

If $m_{I}$ is achieved, then the minimizer is a solution of (1.2) for some $\theta$.

Method II: Consider the minimization problem

$$
m_{I I}=\inf \left\{F_{1}(u)+k F_{3}(u)-\theta F_{4}(u) \mid u \in X, F_{2}(u)=\lambda_{I I}>0\right\} .
$$

If $m_{I I}$ is achieved, then again the minimizer is a solution of (1.2) for some $\mu$.

Method III: Study the minimization problem

$$
m_{I I I}=\inf \left\{F_{5}(u) \mid 0 \neq u \in X, F_{6}(u)=0\right\} .
$$

If $m_{I I I}$ is achieved, then the minimizer is a solution of (1.2).

When $k=0$, the three methods mentioned above are almost equivalent in the sense that they can be changed from one to another by scaling. However, when $k \neq 0$, the term introduced by $F_{3}(u)$ makes the problem substantially different from the case of $k=0$ since $F_{3}$ is nonconvex and the scaling argument does not work. Method III has been used in 11, 12, 13, to study the existence of solutions of (1.2). But it seems that it is too difficult to be used to study orbital stability (see the precise definition in Section 3) of standing waves of (1.1) since $F_{6}$ usually does not satisfy any conservative laws (see Lemma 2.11). Hence our purpose here is to take Method II to study the existence and orbital stability of standing waves of (1.1) under the basic assumption $\inf _{x \in \mathbb{R}^{N}} V(x) \geq 0$ and $\lim _{|x| \rightarrow \infty} V(x)=+\infty$.

We recall that in the case of $k=0$, the existence and orbital stability of a standing wave solution of (1.1) has been studied by Cazenave and Lions [6] via the concentration 
compactness principle. However, the presence of the quasilinear term $\left(\Delta\left(|z|^{2}\right)\right) z$ makes the problem substantially different from the semilinear case. For example, when $k=0$, one can use $\left\{\left.u\left|\int\right| u\right|^{2}=\right.$ const. $\}$ or $\left\{\left.u\left|\int\right| u\right|^{p}=\right.$ const. $\}$ as a constraint to study a corresponding minimization problem. Then the study of orbital stability can proceed after a standard scaling argument. But the term introduced by $\left(\Delta\left(|z|^{2}\right)\right) z$ makes the scaling arguments fail. Hence we need to restrict the arguments to a function space which consists of functions with radial symmetry. The main results are Theorem 3.2 and Theorem 3.4, which are contained in Section 3 after some preliminaries given in Section 2. In Section 4, we study problem (1.1) in one spatial dimension. Some improvements of Theorem 3.2 and Theorem 3.4 are obtained; see Theorem 4.6 and Theorem 4.8

2. Preliminaries. Throughout this paper, all integrals are taken over $\mathbb{R}^{N}$ (or $\mathbb{R}$, depending upon the context) unless stated otherwise. All $d x$ in the integrals are omitted. $o(1)$ will denote a generic infinitesimal as $n \rightarrow \infty$. $\rightarrow$ denotes the strong convergence and $\rightarrow$ the weak convergence. $B(x, R)$ will denote a ball centered at $x$ with radials $R$, and $B(0, R)$ is simply denoted by $B_{R}$. To continue, we list some lemmas which will be useful in what follows. First

Lemma 2.1 (Conservative laws). Let $z(x, t)$ be a solution of (1.1) with initial value $z_{0}$. Then it follows that

$$
\begin{gathered}
\int|z(x, t)|^{2}=\int\left|z_{0}\right|^{2} \equiv \text { const. } \quad \text { (Conservation of "mass"), } \\
\int\left(\frac{1}{2}\left(|\nabla z|^{2}+V|z|^{2}\right)+\left.\left.\frac{k}{4}|\nabla| z\right|^{2}\right|^{2}-\frac{\theta}{p}|z|^{p}\right) \\
=\int\left(\frac{1}{2}\left(\left|\nabla z_{0}\right|^{2}+V\left|z_{0}\right|^{2}\right)+\left.\left.\frac{k}{4}|\nabla| z_{0}\right|^{2}\right|^{2}-\frac{\theta}{p}\left|z_{0}\right|^{p}\right) \\
\equiv \text { const. (Conservation of "energy"). }
\end{gathered}
$$

Proof. This kind of result is known; see e.g. 9].

Lemma $2.2([10])$. Let $\left(\rho_{n}\right)$ be bounded in $L^{1}(\mathbb{R})$. By extracting a subsequence, we may assume that $\left(\rho_{n}\right)$ satisfies one of the following two possibilities:

(i): (Vanishing) $\lim _{n \rightarrow \infty} \sup _{y \in \mathbb{R}} \int_{y+B_{R}} \rho_{n}(x) d x=0$ for all $0<R<+\infty$.

(ii): (Nonvanishing) There exist $\alpha>0, R<+\infty$ and $\left(y_{n}\right) \subset \mathbb{R}$ such that

$$
\lim _{n \rightarrow \infty} \int_{y_{n}+B_{R}} \rho_{n}(x) d x \geq \alpha>0 .
$$

Lemma $2.3([0])$. Let $\left(u_{n}\right)$ be bounded in $H^{1}(\mathbb{R})$. Assume that for some $q>2$ and $R>0$,

$$
\sup _{y \in \mathbb{R}} \int_{y+B_{R}}\left|u_{n}\right|^{q} d x \rightarrow 0 \quad \text { as } \quad n \rightarrow \infty .
$$

Then $u_{n} \rightarrow 0$ in $L^{\beta}(\mathbb{R})$ for any $\beta \geq 2$. 
Lemma 2.4 ([15, Lemma 2 on page 333]). If $u_{n} \rightarrow u$ in $H^{1}(\mathbb{R})$ and $u_{n} \rightarrow u$ a.e. in $\mathbb{R}$, then

$$
\liminf _{n \rightarrow \infty} \int\left|\left(\left|u_{n}\right|^{2}\right)^{\prime}\right|^{2} \geq \int\left|\left(|u|^{2}\right)^{\prime}\right|^{2}+\liminf _{n \rightarrow \infty} \int\left|\left(\left|u_{n}-u\right|^{2}\right)^{\prime}\right|^{2} .
$$

Let $H_{r}^{1}\left(\mathbb{R}^{N}\right)=\left\{u \in H^{1}\left(\mathbb{R}^{N}\right) \mid u(x)=u(|x|)\right\}$ and $H=H_{r}^{1} \cap X$ and $\|u\|^{2}=$ $\int\left(|\nabla u|^{2}+V(x)|u|^{2}+|u|^{2}\right)$. Then we have the following lemma from Strauss [17.

Lemma 2.5. Let $N \geq 2$. Then the following embedding is compact:

$$
H \hookrightarrow L^{q}\left(\mathbb{R}^{N}\right), \quad 2 \leq q<2^{*},
$$

where $2^{*}=\frac{2 N}{N-2}$ for $N \geq 3$ and $\infty$ for $N=2$.

Lemma 2.6 (15, Lemma 13 on page 340]). If $\left(u_{n}\right) \subset H$ are such that $u_{n} \rightarrow u$ in $H^{1}\left(\mathbb{R}^{N}\right)$, then

$$
\left.\left.\liminf _{n \rightarrow \infty} \int|\nabla| u_{n}\right|^{2}\right|^{2} \geq\left.\left.\int|\nabla| u\right|^{2}\right|^{2}
$$

3. The case of $N \geq 2$. In this section, we assume $V(x)=V(|x|)$. We will first follow the line of method II to study the existence of a standing wave of (1.1) in the case of $\theta>0$. Then we will study the orbital stability of the standing wave. Now for any $\lambda>0$ fixed, consider the following minimization problem:

$$
m_{r}=\inf \left\{E(u)=F_{1}(u)+k F_{3}(u)-\theta F_{4}(u) \mid u \in H, F_{2}(u)=\lambda\right\} .
$$

Lemma 3.1. Let $\left(u_{n}\right) \subset H$ be a minimizing sequence of $m_{r}$. If $2<p<2+\frac{4}{N}$, then $\left\|u_{n}\right\|$ and $\left.\left.\int|\nabla| u_{n}\right|^{2}\right|^{2}$ are uniformly bounded with respect to $n$.

Proof. Since $\left(u_{n}\right) \subset H$ is a minimizing sequence of $m_{r}$, i.e.,

$$
m_{r}+o(1)=F_{1}\left(u_{n}\right)+k F_{3}\left(u_{n}\right)-\theta F_{4}\left(u_{n}\right) ; \quad F_{2}\left(u_{n}\right)=\lambda,
$$

by Sobolev and interpolation inequalities, we deduce that for any $u \in H$ and $s=$ $\left(\frac{1}{p}-\frac{1}{2^{*}}\right) /\left(\frac{1}{2}-\frac{1}{2^{*}}\right)$

$$
|u|_{p}^{p} \leq|u|_{2}^{p s}|u|_{2^{*}}^{p(1-s)} \leq M|u|_{2}^{p s}\|u\|^{p(1-s)} .
$$

The Young inequality implies that

$$
|u|_{p}^{p} \leq \varepsilon\|u\|^{2}+A_{\varepsilon}|u|_{2}^{2 p s /(2-p+p s)} .
$$

In here we use the assumption $2<p<2+\frac{4}{N}$. It follows that

$$
m_{r}+1 \geq F_{1}\left(u_{n}\right)+\left.\left.\frac{k}{4} \int|\nabla| u_{n}\right|^{2}\right|^{2}-\frac{\varepsilon}{p}\left\|u_{n}\right\|^{2}-\frac{A_{\varepsilon}}{p}\left|u_{n}\right|_{2}^{2 p s /(2-p+p s)} .
$$

We obtain from choosing $\varepsilon$ small enough and using $\left|u_{n}\right|_{2}^{2}=2 \lambda$ that $m_{r}>-\infty$ and $\left\|u_{n}\right\|$ and $\left.\left.\int|\nabla| u_{n}\right|^{2}\right|^{2}$ are uniformly bounded with respect to $n$.

Theorem 3.2. Suppose $N \geq 2,2<p<2+\frac{4}{N}$ and $k>0$. Then for any fixed $\theta>0$ and $\lambda>0, m_{r}$ is achieved at some $u_{0}$, i.e., $u_{0}(x)=u_{0}(|x|), F_{2}\left(u_{0}\right)=\lambda, F_{1}\left(u_{0}\right)+k F_{3}\left(u_{0}\right)-$ $\theta F_{4}\left(u_{0}\right)=m_{r}$. 
Proof. Let $\left(u_{n}\right) \subset H$ be a minimizing sequence of $m_{r}$. Lemma 3.1 implies that $\left\|u_{n}\right\|$ and $\left.\left.\int|\nabla| u_{n}\right|^{2}\right|^{2}$ are uniformly bounded with respect to $n$. Going if necessary to a subsequence, we can assume that $u_{n} \rightarrow u_{0}$ in $H^{1}\left(\mathbb{R}^{N}\right)$ and $u_{n} \rightarrow u_{0}$ a.e. in $\mathbb{R}^{N}$. The Brezis-Lieb Lemma [3] implies that

$$
F_{1}\left(u_{n}\right)=F_{1}\left(u_{n}-u_{0}\right)+F_{1}\left(u_{0}\right)+o(1) .
$$

Combining this with (3.1), Lemma 2.5 and Lemma 2.6 we have that

$$
\begin{aligned}
m_{r}+o(1) & =F_{1}\left(u_{n}\right)+\left.\left.\frac{k}{4} \int|\nabla| u_{n}\right|^{2}\right|^{2}-\frac{\theta}{p}\left|u_{n}\right|_{p}^{p} \\
& \geq F_{1}\left(u_{0}\right)+k F_{3}\left(u_{0}\right)-\theta F_{4}\left(u_{0}\right)+\lim _{n \rightarrow \infty} F_{1}\left(u_{n}-u_{0}\right) \\
& \geq F_{1}\left(u_{0}\right)+k F_{3}\left(u_{0}\right)-\theta F_{4}\left(u_{0}\right) \geq m_{r} .
\end{aligned}
$$

It follows that $m_{r}=F_{1}\left(u_{0}\right)+k F_{3}\left(u_{0}\right)-\theta F_{4}\left(u_{0}\right)$ and $\lim _{n \rightarrow \infty} F_{1}\left(u_{n}-u_{0}\right)=0$. Thus we obtain from $\left|u_{n}-u_{0}\right|_{2}^{2} \rightarrow 0$ that $\left\|u_{n}-u_{0}\right\| \rightarrow 0$, i.e., $u_{n} \rightarrow u_{0}$ in $H^{1}\left(\mathbb{R}^{N}\right)$.

The method for studying the minimization problem (3.1) is not new, but it has the merit of studying the orbital stability of the standing wave of (1.1) in the spirit of Cazenave and Lions [6]; see also Albert [1]. Roughly speaking, a set of solutions of (1.1) is said to be stable if any solution of (1.1) remains near the set whenever it starts near the set.

Definition 3.3. A set $S \subset H$ is $H$-stable with respect to (1.1) if for any $\varepsilon>0$, there is $\delta>0$ such that for any $z_{0} \in H \cap H^{s}\left(\mathbb{R}^{N}\right)\left(s \geq \frac{N}{2}+7\right)$ with

$$
\inf _{v \in S}\left(\left\|z_{0}-v\right\|+\left.\left|\int\right| \nabla\left|z_{0}\right|^{2}\right|^{2}-\left.\left.\int|\nabla| v\right|^{2}\right|^{2} \mid\right)<\delta
$$

the solution $z(t, x)$ of (2.1) with initial data $z(0, x)=z_{0}$ can be extended to a global solution in $C\left([0, \infty), H^{s}\left(\mathbb{R}^{N}\right)\right)$ and

$$
\sup _{0 \leq t<\infty} \inf _{v \in S}\left(\|z(\cdot, t)-v\|+\left.\left|\int\right| \nabla|z(\cdot, t)|^{2}\right|^{2}-\left.\left.\int|\nabla| v\right|^{2}\right|^{2} \mid\right)<\varepsilon .
$$

Otherwise $S$ is called $H$-unstable.

Now for any $\lambda>0$, we know from Theorem 3.2 that the set of minimizers, denoted by $S_{\lambda}$, of the minimization problem (3.1) is not empty. Then for any $u_{0} \in S_{\lambda},\left.\left.\int|\nabla| u_{0}\right|^{2}\right|^{2}$ is finite. Hence the integral $\int \nabla\left|u_{0}\right|^{2} \nabla\left(u_{0} v\right)$ exists for any $v \in \mathcal{D}\left(\mathbb{R}^{N}\right)$ because

$$
\int\left|u_{0}\right|\left|\nabla u_{0}\right|^{2}+\left|u_{0}\right|^{2}\left|\nabla u_{0}\right| \leq \int\left(1+\left|u_{0}\right|^{2}\right)\left|\nabla u_{0}\right|^{2}+\left|u_{0}\right|^{2}\left(1+\left|\nabla u_{0}\right|^{2}\right)<+\infty .
$$

Therefore, the standard proof of the Ljusternik Theorem on Lagrange multipliers [4] implies that there exist $\gamma$ such that $u_{0}$ is a weak solution of (1.2) for $\mu=-\gamma$. It follows that $z(x, t)=e^{i \mu t} u_{0}(x)$ is a standing wave of (1.1). Thus $e^{i \mu t} u_{0}(\cdot)$ is the orbit of $u_{0}$. Moreover, for any $t \geq 0$, if $u \in S_{\lambda}$, then $e^{i \mu t} u(x) \in S_{\lambda}$. Our orbital stability result is

Theorem 3.4. Assume that $N \geq 2$ and $2<p<2+\frac{4}{N}$. Then $S_{\lambda}$ is $H$-stable with respect to (1.1) in the sense of Definition 3.3 . 
Proof. Suppose the conclusion is false. Then there exists a number $\varepsilon_{0}>0$, such that for any $\frac{1}{n}>0$, there is $\psi_{n} \in H \cap H^{s}\left(\mathbb{R}^{N}\right)\left(s \geq \frac{N}{2}+7\right)$ with

$$
\inf _{g \in S_{\lambda}}\left(\left\|\psi_{n}-g\right\|+\left.\left|\int\right| \nabla\left|\psi_{n}\right|^{2}\right|^{2}-\left.\left.\int|\nabla| g\right|^{2}\right|^{2} \mid\right)<\frac{1}{n}
$$

but

$$
\sup _{t>0} \inf _{g \in S_{\lambda}}\left(\left\|z_{n}(\cdot, t)-g\right\|+\left.\left|\int\right| \nabla\left|z_{n}(\cdot, t)\right|^{2}\right|^{2}-\left.\left.\int|\nabla| g\right|^{2}\right|^{2} \mid\right) \geq \varepsilon_{0},
$$

where $z_{n}(x, t)$ solves (1.1) with $z_{n}(x, 0)=\psi_{n}$. Hence we can pick the first time $t_{n}$ such that

$$
\inf _{g \in S_{\lambda}}\left(\left\|z_{n}\left(\cdot, t_{n}\right)-g\right\|+\left.\left|\int\right| \nabla\left|z_{n}\left(\cdot, t_{n}\right)\right|^{2}\right|^{2}-\left.\left.\int|\nabla| g\right|^{2}\right|^{2} \mid\right)=\varepsilon_{0} .
$$

Then since $\psi_{n} \rightarrow S_{\lambda}$ in $H^{1}\left(\mathbb{R}^{N}\right)$ and $m_{r}=E(g)$ for all $g \in S_{\lambda}$ and $F_{2}(g)=\lambda$, we have $E\left(\psi_{n}\right) \rightarrow m_{r}$ and $F_{2}\left(\psi_{n}\right) \rightarrow \lambda$ as $n \rightarrow \infty$. Thus we can find a sequence $\beta_{n} \rightarrow 1$ such that $F_{2}\left(\beta_{n} \psi_{n}\right)=\lambda$ for all $n$. It follows from Lemma 2.1 that the sequence $q_{n}=\beta_{n} z_{n}\left(\cdot, t_{n}\right)$ satisfies $F_{2}\left(q_{n}\right)=\lambda$ and

$$
\lim _{n \rightarrow \infty} E\left(q_{n}\right)=\lim _{n \rightarrow \infty} E\left(z_{n}\left(\cdot, t_{n}\right)\right)=\lim _{n \rightarrow \infty} E\left(\psi_{n}\right)=m_{r}
$$

and is therefore a minimizing sequence for $m_{r}$. The proof of Theorem 3.2 implies that $q_{n} \rightarrow q_{0}$ strongly in $H^{1}\left(\mathbb{R}^{N}\right)$ and

$$
\left.\left.\lim _{n \rightarrow \infty} \int|\nabla| q_{n}\right|^{2}\right|^{2}=\left.\left.\int|\nabla| q_{0}\right|^{2}\right|^{2} .
$$

Hence $q_{0} \in S_{\lambda}$ and for $n$ large, we have that

$$
\begin{aligned}
\varepsilon_{0} \leq & \left\|z_{n}\left(\cdot, t_{n}\right)-q_{0}\right\|+\left.\left|\int\right| \nabla\left|z_{n}\left(\cdot, t_{n}\right)\right|^{2}\right|^{2}-\left.\left.\int|\nabla| q_{0}\right|^{2}\right|^{2} \mid \\
= & \left|\frac{1}{\beta_{n}}-1\right|\left\|q_{n}\right\|+\left\|q_{n}-q_{0}\right\| \\
& +\left.\left.\left|\frac{1}{\beta_{n}^{4}}-1\right| \int|\nabla| q_{n}\right|^{2}\right|^{2}+\left.\left|\int\right| \nabla\left|q_{n}\right|^{2}\right|^{2}-\left.\left.\int|\nabla| q_{0}\right|^{2}\right|^{2} \mid \rightarrow 0
\end{aligned}
$$

as $n \rightarrow \infty$, which is a contradiction.

REMARK 3.5. Please note that in the stating of this theorem, we have tacitly assumed that the potential $V(x), p$ and the initial data $z_{0}$ belong to a class in which a unique solution of the initial-value problem for (1.1) exists for all $t \in\left[0, T^{*}\right)$ and some $T^{*}<\infty$ or $T^{*}=\infty$. In view of local well-posedness results for this kind of problems studied by Lange-Poppenberg [9] and the arguments developed by Cazenave [5], we will continue to make these assumptions without any comments. Moreover, if $z_{0}$ is radially symmetrical with respect to $x$, then so is $z(x, t)$.

REmark 3.6. Note that in Theorem 3.2 and Theorem 3.4. $V(x)$ can cover the case of $V(x)=|x|^{2}$. Problem (1.1) in the case of $V(x)=|x|^{2}$ and $k=0$ describes the BoseEinstein condensate with attractive interparticle interactions under a magnetic trap as the Gross-Pitaevski equation with a harmonic potential term [18]. Hence Theorem 3.4 generalizes [19, Theorem 3.2]. 
Remark 3.7. As pointed out in [1, page 4], although the Concentration Compactness method for proving the orbital stability of a standing wave has the advantage of requiring less analysis than others (see, e.g., [7] and the references therein), it produces a weak result in that it only demonstrates stability of a set of minimizing solutions without providing information on the structure of the set, or distinguishing among its different members. This kind of possible confusion will be clarified in the case of $N=1$.

4. The case of $N=1$. In this section, we will give a stronger orbital stability of the standing wave of problem (1.1) in the case of $V(x) \equiv 0$ and $N=1, k \geq 0, \theta>0$. At this time, we write (1.1) as

$$
i \partial_{t} z=-z^{\prime \prime}-k\left(|z|^{2}\right)^{\prime \prime} z-\theta|z|^{p-2} z
$$

and denote by " (resp. ') the second (resp. first) order spatial derivatives. Finding a standing wave of (4.1) of the form $e^{i \mu t} u(x)$ is equivalent to solving the associated elliptic problem

$$
-u^{\prime \prime}+\mu u-k\left(\left(|u|^{2}\right)^{\prime \prime}\right) u-\theta|u|^{p-2} u=0 .
$$

Using the basic function space $H^{1}(\mathbb{R})=W^{1,2}(\mathbb{R})$ with the standard norm $\|u\|^{2}=$ $\int\left(|\nabla u|^{2}+|u|^{2}\right)$ and the continuous embedding $H^{1}(\mathbb{R}) \hookrightarrow L^{\infty}(\mathbb{R})$, we know that the functional

$$
I(u)=\int\left(\frac{1}{2}\left|u^{\prime}\right|^{2}+\frac{k}{4}\left|\left(|u|^{2}\right)^{\prime}\right|^{2}-\frac{\theta}{p}|u|^{p}\right)
$$

is well defined on $H^{1}(\mathbb{R})$. Moreover, we have

Lemma 4.1 ([15, Lemma 1]). $I$ is of class $C^{1}$ on $H^{1}(\mathbb{R})$.

For any $\lambda>0$ fixed, we define

$$
\mathcal{M}=\left\{\left.u \in H^{1}(\mathbb{R})\left|\frac{1}{2} \int_{\mathbb{R}}\right| u\right|^{2}=\lambda\right\}, \quad m=\inf _{u \in \mathcal{M}} I(u) .
$$

Lemma 4.2. If $2<p<6$, then $-\infty<m<0$.

Proof. Suppose $\psi(x) \in \mathcal{M}$. Then so is $\xi^{\frac{1}{2}} \psi(\xi x)$ for $\xi>0$. Hence

$$
\begin{aligned}
I\left(\xi^{\frac{1}{2}} \psi(\xi x)\right) & =\int_{\mathbb{R}}\left(\frac{1}{2} \xi^{3}\left|\psi^{\prime}(\xi x)\right|^{2}+\frac{k}{4} \xi^{4}\left|\left(|\psi(\xi x)|^{2}\right)^{\prime}\right|^{2}-\frac{\theta}{p} \xi^{\frac{p}{2}}|\psi(\xi x)|^{p}\right) \\
& =\int\left(\frac{1}{2} \xi^{2}\left|\psi^{\prime}\right|^{2}+\frac{k}{4} \xi^{3}\left|\left(|\psi|^{2}\right)^{\prime}\right|^{2}-\frac{\theta}{p} \xi^{\frac{p}{2}-1}|\psi|^{p}\right) .
\end{aligned}
$$

It follows from $2<p<6$ and $m \leq I\left(\xi^{\frac{1}{2}} \psi(\xi x)\right)$ that $m<0$ provided $\xi>0$ small enough. Now for any $u \in \mathcal{M}$, we can use Sobolev and interpolation inequalities such that for some $0<\alpha<1$,

$$
|u|_{p}^{p} \leq|u|_{2}^{p \alpha}|u|_{\infty}^{p(1-\alpha)} \leq d|u|_{2}^{p \alpha}\|u\|^{p(1-\alpha)} .
$$

The Young inequality implies that

$$
|u|_{p}^{p}=\varepsilon\|u\|^{2}+A_{\varepsilon}|u|_{2}^{2 p \alpha /(2-p+p \alpha)} .
$$


In here, we use the assumption $2<p<6$. By choosing some $u \in \mathcal{M}$ such that

$$
m+1 \geq \frac{1}{2}\|u\|^{2}-\lambda+\frac{k}{4} \int_{\mathbb{R}}\left|\left(|u|^{2}\right)^{\prime}\right|^{2}-\frac{\theta}{p} \varepsilon\|u\|^{2}-\frac{\theta}{p} A_{\varepsilon}|u|_{2}^{2 p \alpha /(2-p+p \alpha)}
$$

and $\varepsilon$ small enough, we can get that $m>-\infty$.

Lemma 4.3. Let $\left\{u_{n}\right\}$ be a minimizing sequence of $m$. Then there exist $w \neq 0$ and $\left(y_{n}\right) \subset \mathbb{R}$ such that $u_{n}\left(\cdot+y_{n}\right) \rightarrow w$ in $H^{1}(\mathbb{R})$.

Proof. Let $\left\{u_{n}\right\}$ be a minimizing sequence of $m$. Then from the proof of the second part of Lemma 4.2, we know that $\left(u_{n}\right)$ is bounded in $H^{1}(\mathbb{R})$. Using the fact that $\frac{1}{2} \int_{\mathbb{R}}\left|u_{n}\right|^{2}=\lambda>0$ and Lemma 2.3, we know that the vanishing case does not occur for $\rho_{n}(x)=\left|u_{n}(x)\right|^{2}$. Therefore, there exist $\varepsilon>0$ and $R>0$ such that

$$
\liminf _{n \rightarrow \infty} \sup _{y \in \mathbb{R}} \int_{y-R}^{y+R}\left|u_{n}(x)\right|^{2} \geq \varepsilon>0 .
$$

We may assume that there are $\left(y_{n}\right) \subset \mathbb{R}$ and $R>0$ such that

$$
\liminf _{n \rightarrow \infty} \int_{y_{n}-R}^{y_{n}+R}\left|u_{n}(x)\right|^{2} \geq \frac{\varepsilon}{2}>0 .
$$

Hence $w_{n}(x)=u_{n}\left(x+y_{n}\right)$ satisfies $I\left(w_{n}\right)=I\left(u_{n}\right)$ and $\frac{1}{2} \int_{\mathbb{R}}\left|w_{n}\right|^{2}=\lambda$; i.e., the sequence $\left(w_{n}\right)$ is also a minimizing sequence which satisfies

$$
\liminf _{n \rightarrow \infty} \int_{-R}^{R}\left|w_{n}(x)\right|^{2} \geq \frac{\varepsilon}{2}>0 .
$$

$\left(w_{n}\right)$ is also bounded in $H^{1}(\mathbb{R})$. Going if necessary to a subsequence, we have $w_{n} \rightarrow w$ in $H^{1}(\mathbb{R})$ and thus $w_{n} \rightarrow w$ in $L_{l o c}^{2}(\mathbb{R})$. (4.10) implies that $w \neq 0$.

Lemma 4.4. Let $w_{n} \rightarrow w$ in $H^{1}(\mathbb{R})$ and $w_{n} \rightarrow w$ a.e. in $\mathbb{R}$. Then

$$
\begin{gathered}
\lim _{n \rightarrow \infty}\left(\left|w_{n}\right|_{q}^{q}-\left|w_{n}-w\right|_{q}^{q}\right)=|w|_{q}^{q}, \quad q \geq 2 ; \\
\lim _{n \rightarrow \infty}\left(\left|w_{n}^{\prime}\right|_{2}^{2}-\left|\left(w_{n}-w\right)^{\prime}\right|_{2}^{2}\right)=\left|w^{\prime}\right|_{2}^{2} .
\end{gathered}
$$

Proof. These are direct consequence of the Brezis-Lieb Lemma 3 .

Proposition 4.5. Suppose $4 \leq p<6$. Then $m$ is achieved by some nonnegative $w$; i.e., there is $0 \neq w \in \mathcal{M}$ with $I(w)=m$ and $w \geq 0$.

Proof. Let $\left(u_{n}\right)$ be a minimizing sequence of $m$. Lemma 4.3 implies that there are $\left(y_{n}\right)$ and $w \neq 0$ such that $w_{n}(x)=u_{n}\left(x+y_{n}\right)$ and $w_{n} \rightarrow w \neq 0$ in $H^{1}(\mathbb{R})$. To conclude the proof, we have to show that $w \in \mathcal{M}$, i.e., $\frac{1}{2}|w|_{2}^{2}=\lambda$.

Arguing by a contradiction, we may assume $0<\frac{1}{2}|w|_{2}^{2}<\lambda$. For $v_{n}=w_{n}-w$, we may assume from Lemma 4.4 that $\frac{1}{2}\left|v_{n}\right|_{2}^{2}<\lambda$. Denote

$$
\tilde{w}=w /\left(\frac{1}{2 \lambda}\right)^{\frac{1}{2}}|w|_{2} \quad \text { and } \quad \tilde{v}_{n}=v_{n} /\left(\frac{1}{2 \lambda}\right)^{\frac{1}{2}}\left|v_{n}\right|_{2} .
$$


We have that

$$
\begin{aligned}
m & \geq \int_{\mathbb{R}}\left(\frac{1}{2}\left|w_{n}^{\prime}\right|^{2}+\frac{k}{4}\left|\left(\left|w_{n}\right|^{2}\right)^{\prime}\right|^{2}-\frac{\theta}{p}\left|w_{n}\right|^{p}\right) \\
& \geq \int_{\mathbb{R}}\left(\frac{1}{2}\left|w^{\prime}\right|^{2}+\frac{k}{4}\left|\left(|w|^{2}\right)^{\prime}\right|^{2}-\frac{\theta}{p}|w|^{p}\right) \\
& +\int_{\mathbb{R}}\left(\frac{1}{2}\left|v_{n}^{\prime}\right|^{2}-\frac{\theta}{p}\left|v_{n}\right|^{p}\right)+\frac{k}{4} \liminf _{n \rightarrow \infty} \int_{\mathbb{R}}\left|\left(\left|v_{n}\right|^{2}\right)^{\prime}\right|^{2} \\
& =\left(\sqrt{\frac{1}{2 \lambda}}|w|_{2}\right)^{2} \int_{\mathbb{R}} \frac{1}{2}\left|\tilde{w}^{\prime}\right|^{2}+\frac{k}{4}\left(\sqrt{\frac{1}{2 \lambda}}|w|_{2}\right)^{4} \int_{\mathbb{R}}\left|\left(|\tilde{w}|^{2}\right)^{\prime}\right|^{2} \\
& -\left(\sqrt{\frac{1}{2 \lambda}}|w|_{2}\right)^{p} \frac{\theta}{p} \int_{\mathbb{R}}|\tilde{w}|^{p}+\left(\sqrt{\frac{1}{2 \lambda}}\left|v_{n}\right|_{2}\right)^{2} \int_{\mathbb{R}} \frac{1}{2}\left|\tilde{v}_{n}^{\prime}\right|^{2} \\
& -\left(\sqrt{\frac{1}{2 \lambda}}\left|v_{n}\right|_{2}\right)^{p} \frac{\theta}{p} \int_{\mathbb{R}}\left|\tilde{v}_{n}\right|^{p}+\frac{k}{4} \liminf \left(\sqrt{\frac{1}{2 \lambda}}\left|v_{n}\right|_{2}\right)^{4} \int_{\mathbb{R}}\left|\left(\left|\tilde{v}_{n}\right|^{2}\right)^{\prime}\right|^{2} \\
& >\left(\sqrt{\frac{1}{2 \lambda}}|w|_{2}\right)^{4} \int_{\mathbb{R}}\left(\frac{1}{2}\left|\tilde{w}^{\prime}\right|^{2}+\frac{k}{4}\left|\left(|\tilde{w}|^{2}\right)^{\prime}\right|^{2}-\frac{\theta}{p}|\tilde{w}|^{p}\right)^{2} \quad(\text { since } p \geq 4) \\
& +\liminf _{n \rightarrow \infty}\left(\sqrt{\frac{1}{2 \lambda}}\left|v_{n}\right|_{2}\right)^{4} \int_{\mathbb{R}}\left(\frac{1}{2}\left|\tilde{v}_{n}^{\prime}\right|^{2}+\frac{k}{4}\left|\left(\left|\tilde{v}_{n}\right|^{2}\right)^{\prime}\right|^{2}-\frac{\theta}{p}\left|\tilde{v}_{n}\right|^{p}\right) \\
& =\left(\sqrt{\frac{1}{2 \lambda}}|w|_{2}\right)^{4} I(w)+\liminf _{n \rightarrow \infty}\left(\sqrt{\frac{1}{2 \lambda}}\left|v_{n}\right|_{2}\right)^{4} I\left(\tilde{v}_{n}\right) \\
& \geq m\left(\left(\sqrt{\frac{1}{2 \lambda}}|w|_{2}\right)^{4}+\liminf _{n \rightarrow \infty}\left(\sqrt{\frac{1}{2 \lambda}}\left|v_{n}\right|_{2}\right)^{4}\right) \\
& \geq m\left(\left(\sqrt{\frac{1}{2 \lambda}}|w|_{2}\right)^{2}+\liminf _{n \rightarrow \infty}\left(\sqrt{\frac{1}{2 \lambda}}\left|v_{n}\right|_{2}\right)^{2}\right) \quad(\text { since } m<0) \\
& =m,
\end{aligned}
$$

which is a contradiction. In here the last equality follows from using Lemma 4.4 and $\frac{1}{2}\left|w_{n}\right|_{2}^{2}=\lambda$. Up to now we have proved that $\frac{1}{2}|w|_{2}^{2}=\lambda$, i.e., $w_{n} \rightarrow w$ in $L^{2}(\mathbb{R})$. Since $\left(w_{n}\right)$ is bounded in $H^{1}(\mathbb{R})$, the standard interpolation and Sobolev inequalities imply that $w_{n} \rightarrow w$ in $L^{p}(\mathbb{R})$. We now obtain from $\left(w_{n}\right)$ being a minimizing sequence and Lemma 2.4 that

$$
\begin{aligned}
m & \geq \int_{\mathbb{R}}\left(\frac{1}{2}\left|w_{n}^{\prime}\right|^{2}+\frac{k}{4}\left|\left(\left|w_{n}\right|^{2}\right)^{\prime}\right|^{2}-\frac{\theta}{p}\left|w_{n}\right|^{p}\right) \\
& \geq \int_{\mathbb{R}}\left(\frac{1}{2}\left|w^{\prime}\right|^{2}+\frac{k}{4}\left|\left(|w|^{2}\right)^{\prime}\right|^{2}-\frac{\theta}{p}|w|^{p}\right)+\lim _{n \rightarrow \infty} \int_{\mathbb{R}} \frac{1}{2}\left|\left(w_{n}-w\right)^{\prime}\right|^{2} \\
& \geq m+\lim _{n \rightarrow \infty} \int_{\mathbb{R}} \frac{1}{2}\left|\left(w_{n}-w\right)^{\prime}\right|^{2} .
\end{aligned}
$$

Hence $\int_{\mathbb{R}}\left|\left(w_{n}-w\right)^{\prime}\right|^{2} \rightarrow 0$. Combining this with $w_{n} \rightarrow w$ in $L^{2}(\mathbb{R})$ we get that $w_{n} \rightarrow w$ in $H^{1}(\mathbb{R})$. Note that if $\left(w_{n}\right)$ is a minimizing sequence of $m$, then so is $\left(\left|w_{n}\right|\right)$. Therefore the conclusion follows. 
Theorem 4.6. Assume $4 \leq p<6$. Then there exists $\gamma<0$ such that (4.2) admits a positive solution $u \in H_{0}^{1}(\mathbb{R})$ for any $\theta>0$ and $\mu=-\gamma$.

Proof. With the help of Proposition 4.5 and an argument in front of Theorem 3.4, we know that there exists a nonnegative $u \in H^{1}(\mathbb{R})$ with $|u|_{2}^{2}=2 \lambda$ and a Lagrange multiplier $\gamma \in \mathbb{R}$ such that

$$
-\int u^{\prime \prime} v-k \int\left(u^{2}\right)^{\prime \prime} u v-\theta|u|^{p-2} u v=\gamma \int u v,
$$

for all $v \in H^{1}(\mathbb{R})$. Putting $v=u$ in (4.15), we get that

$$
\gamma|u|_{2}^{2}=\left|u^{\prime}\right|_{2}^{2}+k \int\left|\left(|u|^{2}\right)^{\prime}\right|^{2}-\theta|u|_{p}^{p}
$$

It follows from $0>m=\frac{1}{2}\left|u^{\prime}\right|_{2}^{2}+\frac{k}{4} \int\left|\left(|u|^{2}\right)^{\prime}\right|^{2}-\frac{\theta}{p}|u|_{p}^{p}$ and $p \geq 4$ that $\gamma<0$. Hence $u \in H_{0}^{1}(\mathbb{R})$ is a nonnegative solution of (4.2) for any $\mu=-\gamma$. The proof of positivity of $u$ is the same as [15, Theorem 1] and is omitted here. The proof is completed.

Just as was pointed out in Remark 3.5. we need to use the following basic assumption to study the orbital stability.

(A) For some $p \geq 4, k>0$ and some $z_{0}$, the Cauchy problem (4.1) with initial value $z(x, 0)=z_{0} \in H^{s}(\mathbb{R})\left(s \geq \frac{1}{2}+7\right)$ has a solution $z(x, t)$ in $\left[0, T^{*}\right)$ with $T^{*}<\infty$ or $T^{*}=\infty$.

Denote by $\mathcal{S}_{\lambda}$ the set of minimizers of problem (4.4). Then with the help of Lemma 4.3 and Proposition 4.5, we can check in detail the proof of Theorem 3.4 and get directly the following orbital stability result.

Proposition 4.7. Suppose $(A)$ holds and $4 \leq p<6, \theta>0$. Then $\mathcal{S}_{\lambda}$ is $H$-stable with respect to (4.1) in the sense of Definition 3.3.

We are now in a position to state the following stronger orbital stability result.

Theorem 4.8. Suppose $(A)$ holds and $4 \leq p<6, \theta>0$. Then for any $\varepsilon>0$, there exists $\delta>0$ such that if $z_{0} \in H^{s}(\mathbb{R})$ and

$$
\inf _{\eta, \xi \in \mathbb{R}}\left(\left\|z_{0}-e^{i \eta} u_{0}(\cdot+\xi)\right\|+\left.\left|\int\right| \nabla\left|z_{0}\right|^{2}\right|^{2}-\left.\left.\int|\nabla| u_{0}\right|^{2}\right|^{2} \mid\right)<\delta
$$

then the solution $z(x, t)$ of (4.1) with initial value $z(x, 0)=z_{0}$ can be extended to a global solution and for all existence times $t$, it follows that

$$
\inf _{\eta, \xi \in \mathbb{R}}\left(\left\|z(\cdot, t)-e^{i \eta} u_{0}(\cdot+\xi)\right\|+\left.\left|\int\right| \nabla|z|^{2}\right|^{2}-\left.\left.\int|\nabla| u_{0}\right|^{2}\right|^{2} \mid\right)<\varepsilon .
$$

Proof. It suffices to prove that $\mathcal{S}_{\lambda}=\left\{e^{i \eta} u_{0}(x+\xi)\right\}$. If $u \in \mathcal{S}_{\lambda}$, then $e^{i \eta} u(x) \in \mathcal{S}_{\lambda}$ and an argument presented in the proof of Theorem 4.6 implies that $u$ being a solution of (4.2) with some $\mu$. Note that $u(x)$ being a positive solution of (4.2) is equivalent to $w(x)=\theta^{\frac{1}{p-2}} \mu^{\frac{1}{2-p}} u\left(\mu^{-\frac{1}{2}} x\right)$ being a solution of

$$
-w^{\prime \prime}+w-k \theta^{\frac{2}{2-p}} \mu^{\frac{1}{p-2}}\left(w^{2}\right)^{\prime \prime} w=w^{p-1}, \quad w>0, \quad w \in H^{1}(\mathbb{R}) .
$$


By a result of Ambrosetti-Wang [2], we know that (4.18) has a unique positive solution $w_{0}(x)$ up to a translation. It follows that $u_{0}(x)=\theta^{\frac{1}{p-2}} \mu^{\frac{1}{p-2}} w_{0}\left(\mu^{\frac{1}{2}} x\right)$ is the unique positive solution of (4.2) up to a translation. The proof is complete.

\section{REFERENCES}

[1] J. P. Albert, Concentration compactness and the stability of solitary-wave solutions to nonlocal equations, Contemporary Mathematics 221 (1999) 1-29. MR.1647189 (99m:35199)

[2] A. Ambrosetti and Z.Q. Wang, Positive solutions to a class of quasilinear elliptic equations on $\mathbb{R}$, Disc. Contin. Dynam. Syst. 9 (2003), 55-68. MR.1951313 (2003m:34050)

[3] H. Brezis and E. Lieb, A relation between pointwise convergence of functions and convergence of functionals, Proc. Amer. Math. Soc. 88 (1983) 486-490. MR699419 (84e:28003)

[4] F.E. Browder, Variational methods for nonlinear elliptic eigenvalue problems, Bull. Amer. Math. Soc. 71 (1965) 176-183. MR.0179459 (31:3707)

[5] T. Cazenave, Stable solutions of the logarithmic Schrödinger equation, Nonlinear Anal. TMA 7 (1983) 1127-1140. MR.719365 (84m:35102)

[6] T. Cazenave and P. L. Lions, Orbital stability of standing waves for some nonlinear Schrödinger equations, Comm. Math. Phys. 85 (1982) 549-561. MR677997 (84i:81015)

[7] M. Grillakis, J. Shatah and W. Strauss, Stability theory of solitary waves in the presence of symmetry I, J. Functional Analysis 74 (1987), 160-197; II, J. Functional Analysis 94 (1990) 308-348. MR.901236 (88g:35169)

[8] S. Kurihura, Large amplitude quasi-solitons in superfluid film, J. Phys. Soc. Japan 50 (1981) 32623267.

[9] H. Lange, M. Poppenberg and H. Teismann, Nash-Moser methods for the solution of quasilinear Schrödinger equations, Comm. Partial Diff. Equations 24 (1999) 1399-1418. MR1697492 (2000e:35212)

[10] P.L. Lions, The concentration-compactness principle in the calculus of variations, The locally compact case, Parts 1 and 2, Ann. Inst. Henri Poincaré Anal. Non Linéaire 1 (1984), 109-145; 223-283. MR778970 (87e:49035a) MR778974(87e:49035b)

[11] J. Q. Liu and Z. Q. Wang, Soliton solutions for quasilinear Schrödinger equations, I, Proc. Amer. Math. Soc. 131 (2003) 441-448. MR.1933335(2003k:35232)

[12] J. Q. Liu, Y. Wang and Z. Q. Wang, Soliton solutions for quasilinear Schrödinger equations, II, J. Differential Equations 187 (2003) 473-493. MR1949452 (2004e:35074)

[13] J. Q. Liu, Y. Wang and Z. Q. Wang, Solutions for quasilinear Schrödinger equations via the Nehari method, Comm. Partial Diff. Equations 29 (2004) 879-901. MR.2059151 (2005d:35080)

[14] A. Nakamura, Damping and modification of exciton solitary waves, J. Phys. Soc. Japan 42 (1977) 1824-1835.

[15] M. Poppenberg, K. Schmitt and Z. Q. Wang, On the existence of soliton solutions to quasilinear Schrödinger equations, Cal. Var. and PDEs 14 (2002) 329-344. MR.1899450 (2003d:35247)

[16] P. Rabinowitz, On a class of nonlinear Schrödinger equations, Z. Angew. Math. Phys. 43 (1992) 270-291. MR 1162728 (93h:35194)

[17] W. Strauss, Existence of solitary waves in higher dimensions, Comm. Math. Phys. 55 (1977) 149162. MR0454365 (56:12616)

[18] T. Tsurumi and M. Wadati, Collapses of wavefunctions in multi-dimensional nonlinear Schrödinger equations under harmonic potential, J. Phys. Soc. Japan 66(1997) 1-8.

[19] J. Zhang, Stability of standing waves for nonlinear Schrödinger equations with unbounded potentials, Z. Angew. Math. Phys. 51 (2000) 498-503. MR1762704 (2001c:35227) 